\title{
Isonousía y pereza en el pensamiento de Jacques Rancière
}

\author{
Isonousia and sloth in Jacques Rancière's thought
}

\author{
RAQUEL FERRÁNDEZ-FORMOSO*
}

\begin{abstract}
Resumen: La pedagogía es un tema crucial en el pensamiento de Rancière y juega un rol principal en su obra El maestro ignorante, texto que el presente escrito analiza y cuestiona. Su propuesta emancipadora establece como punto de partida una igualdad de inteligencias que en este escrito hemos denominado «isonousía», y según nuestra hipótesis, esta igualdad lleva aparejada una desigualdad de las voluntades (anisothelema). En consecuencia, la propuesta de Rancière no altera en absoluto "el orden explicador" que denuncia, pues el maestro emancipador ya no tiene que corregir la ignorancia, pero sí la pereza; no ha de impartir un conocimiento superior, pero sí imponer una voluntad superior.

Palabras clave: nous, thelema, pereza, monotecnia, explicación, maestro, pedagogía, educación, voluntad, inteligencia.
\end{abstract}

\begin{abstract}
Pedagogy is a crucial issue in Rancière's philosophy and it plays a main role in his famous work The ignorant schoolmaster, that this paper analyses and calls into question. His emancipatory proposal establishes as a starting point an equality of intelligences which in this paper will be called «isonousía», and according to our hypothesis, this equality involves the inequality of the wills (anisothelema). Conquently, Rancière's proposal does not alter at all the «explicative order» that it denounces, since the emancipating master no longer has to correct ignorance, but sloth does; her role does not consist of imparting a superior knowledge, but of imposing a higher will.
\end{abstract}

Keywords: nous, thelema, sloth, monotechnic, pedagogy, education, will, intelligence.

\section{Introducción}

La continuidad del pensamiento de Rancière hace de la diversidad de sus obras -estéticas, pedagógicas, políticas- distintas tonalidades de un mismo cuadro formando parte de un lenguaje que, a través del método de la igualdad, puede ser enseñado incluso sin antes haber sido aprendido. Tanto "la pintura, como la escultura, el grabado o cualquier otro arte es un lenguaje que puede ser entendido, hablado por cualquiera que tenga la inteligencia de su propio lenguaje" (Rancière, 2003: 39). A esto habría que sumar el resto de artes propiamente discursivas, como la política o la filosofía. Sea cual sea la materia artística, se trata de tomar

Recibido: 25/04/2018. Aceptado: 23/05/2019.

* Doctoranda en Filosofía en la Universidad Nacional de Educación a Distancia (UNED), mi área principal de investigación es la Filosofía india. Entre mis publicaciones recientes, se cuentan: "La presencia mística de Lalla: poeta y yoguini śivaíta del siglo XIV d.C.", Aposta. Revista de ciencias sociales; "Ser y tiempo en Nietzsche. El legado hermenéutico de Martin Heidegger”, Metafísica y Persona. Email: ferrandez.raquel@ gmail.com 
conciencia de la igual capacidad de todos los seres humanos de aprender y comprender "el querer decir de la obra" (Rancière, 2003: 23). Incluso cuando la forma natural de esta obra, como apuntó Derrida, no sea el decir (2001: 33)1. En Rancière, no obstante, la racionalidad (de la voluntad, de la igualdad, del arte, de la política) es siempre discursiva, y aprehensible discursivamente, independientemente de la forma bajo la que se exprese. La igualdad de las inteligencias es la igualdad de los sujetos hablantes, pero sobre todo, de los sujetos que tienen la misma capacidad, no ya para decir el logos, sino para el logos mismo. En el proceso de emancipación, estos sujetos se vuelven conscientes de su propia potencia y conscientes, también, de la arbitrariedad del lenguaje. El fonocentrismo que Rancière denuncia en Platón ${ }^{2}$, se ve compensado en su propia obra por un logocentrismo que liga cualquiera de las partes -cualquiera de los conceptos, de los puntos de partida posibles- al todo, pero siempre bajo el velo de la racionalidad discursiva. Tal y como Jacotot propone en su método de enseñanza universal, es preciso tomar un punto cualquiera de referencia y relacionarlo con todo lo demás, dado que "todo está en todo". En el pensamiento de Rancière encontramos trazados, de un modo parecido, los rasgos de una problemática continua, de una continuidad politemática. Estas preocupaciones constantes con las que lidia en todas sus obras, son acompañadas muchas veces por las mismas referencias bibliográficas ${ }^{3}$, contra las que una y otra vez vuelve a batirse, y con la ayuda de las cuales, teje la triple trama política-estética-pedagógica de su pensamiento. Por la puesta en práctica del método pedagógico de la igualdad, quedaría patente la emancipación de los seres humanos en el momento en que descubren que comparten con el resto las mismas capacidades intelectuales. A este principio de la igualdad de las inteligencias, que Rancière presupone como punto de partida, lo llamaremos "isonousía" (iso: igual; nous: inteligencia) en el contexto de este escrito. La isounosía implica invertir la lógica pedagógica de las instituciones educativas, fundada en la desigualdad intelectiva. El objetivo de este escrito es demostrar que la propuesta de Rancière, basada en el modelo educativo de Joseph Jacotot, consiste en presuponer la igualdad de las inteligencias para reforzar otra clase de desigualdad, la de las voluntades (anisothelema). El maestro emancipado ha de ser un pedagogo intratable e imponer su voluntad a la voluntad de sus alumnos. Por un lado, el orden equitativo de Rancière y el "orden explicador" que denuncia comparten el mismo enemigo: la pereza. Por otro lado, en el método pedagógico de Rancière encontramos no solo una ausencia total de los afectos en materia pedagógica, sino que éstos parecen ser incluso contraproducentes para la tarea del maestro. En consecuencia, la jerarquía de las inteligencias que Rancière denuncia en el modelo político de Platón, no se ve necesariamente alterada con un método que simplemente refuerza la equidad intelectiva para fortalecer la desigualdad volitiva, saltándose las bases de cualquier educación emocional. En las páginas que siguen, analizaremos las diferencias y similitudes

1 "Así, se pregunta lo que quiere decir una obra plástica o musical sometiendo todas las producciones a la autoridad de la palabra y de las artes “discursivas”. Derrida (2001) La verdad en pintura. Barcelona: Paidós, p. 33.

2 Rancière (2003). El maestro ignorante. Barcelona: Laertes. P. 24.; J.Rancière denuncia la oposición socrática entre la palabra viva y la letra muerta plasmada en Fedro (274c - 277a). No obstante, esta condena platónica de la escritura no es total e irreversible, tal y como Rancière la presenta. En Fedro (275d) Sócrates considera a la logografía como un buen método para recordar lo que ya sabemos. Por el contrario, Sócrates se opone a quienes conciben la escritura como un método de aprendizaje.

3 Cfr. El pasaje de Sócrates y el esclavo, de corte eminentemente pedagógico, puede encontrarse citado en: $E l$ filósofo y los pobres (p. 64), El maestro ignorante (p.20), El desacuerdo (p.32). Todas estas obras están citadas en el apartado final de referencias. 
entre la pereza de los gobernantes y la de los gobernados, la del maestro explicador y la del alumno embrutecido, y en fin, la pereza que nos concierne de manera más inmediata, la del creador y la del espectador, la de quien escribe estas líneas y la de quien las lee.

\section{La pereza de Platón}

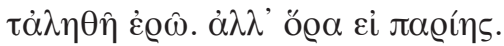

Voy a decir la verdad. ¿Crees que lo permitirás?

Platón, Banquete (214e)

En su particular interpretación del diálogo La República de Platón, Jacques Rancière ${ }^{4}$ nos sitúa delante de un Sócrates que hace descansar la armonía de la ciudad ideal en la regla de la monotecnia. Salvaguardar a la polis de la politecnia de los individuos, es decir, asegurarse de que cada ciudadano se adhiere estrictamente a su rol en el marco social y lleva a cabo nada más que su función, es una especie de fundamento obsesivo que recorre todo el diálogo. La división del trabajo debe ser absolutamente respetada. Corresponde al filósoforey el seleccionar las aptitudes naturales más apropiadas a cada individuo, a pesar de que este soberano, nos dice Rancière, es "un hijo del lujo" (1983: 33) y por lo tanto, un hijo de lo superfluo. Su figura se hace necesaria en el momento en el que el lujo se introduce en la ciudad y con él, la guerra en tanto profesión se modeliza como una necesidad de todo el conjunto social y como una posible amenaza para el mismo. El sector militar debe tomar su modelo de vida de la simplicidad de los artesanos y comerciantes, es decir, debe limitarse a cumplir con su función y satisfacerse con esta mono-tarea.

La especialización del artesano es ahora ésta: la simple obligación de hacer otra cosa. Toda técnica lleva en sí misma la capacidad de hacer otra cosa. Esta contradicción "politécnica", inherente a la técnica, debe ser corregida constantemente por la regla social: es artesano fabricante aquel al cual le son prohibidos el juego, la mentira, la apariencia. ${ }^{5}$ (Rancière, 1983: 36)

No obstante, el "guerrero" es el sujeto social más propenso a quebrantar esta regla y, por ello, requiere de una educación especial que solo el filósofo-rey puede garantizarle. "La virtud del guerrero es la de volver necesario al filósofo" (Rancière, 1983: 33). A ojos de Rancière, el filósofo-rey propuesto en La República es "un ingeniero de almas" (1983: 38), seleccionador de talentos, naturalezas y aptitudes, y el único que, en el seno de la ciudad platónica, tiene derecho a la mentira.

El ingeniero de almas instaurará, entonces, la mentira necesaria y suficiente; el axioma, el principio indemostrable que parece ser el fin de su obra: la naturaleza. Por

4 Esta interpretación puede encontrarse en: Rancière, Jacques (1983). Le philosophe et ses pauvres. Paris: Fayard. Pp 16-85; Sobre la monotecnia en La República de Platón véase también J. Rancière (2010), El Espectador Emancipado, Buenos Aires: Manantial. P. 26.

5 Traducción propia. 
una contra-técnica, encuentra el medio de hacer creer a la ciudad una noble mentira: es decir una mentira sobre la nobleza, una genealogía a la manera de los poetas $[\ldots]^{6}$ (Rancière, 1983: 38)

El relato de las tres clases de almas (el mito de los tres metales) es una mentira legítima en boca del soberano cuya alma, como sabemos, ha sido forjada en oro. A la luz de esta base interpretativa, Rancière expone conclusiones mucho más complejas sacadas de su lectura del diálogo y que remiten a nuestros regímenes políticos actuales, pasando por una redefinición del comunismo a la luz de La República. Pero hay algo sobre lo que Rancière enseguida rectifica. El rey-soberano no puede ser el único que ostenta el derecho a la mentira. Si se quiere frenar la posible diversidad de talentos que puede reunir un mismo individuo, los artesanos y los comerciantes -las almas de bronce- deben tener acceso a una parte de ese derecho. La restitución de la legítima apariencia les viene dada por medio de la pereza: esta condenable falta de voluntad es la que puede garantizar la monotecnia entre las "naturalezas inferiores", y por tanto, se vuelve "un mal necesario", del mismo modo que la mentira mitológica del rey-ingeniero se torna legítima y comprensible en razón de los fines que persigue.

La pereza y la incompetencia son las disposiciones más propicias para asegurar la única cosa importante: que el artesano no lleve a cabo más que una sola tarea, aquella que le sirve de señal para colocarlo en su lugar. Porque aquel que es experto en arreglar sus zapatos podría serlo también en coser su abrigo, trenzar su cinturón y forjar sus anillos. ¿Y entonces, por qué no iba a convertirse también en fabricante de discursos y mercader de sabiduría?7 (Rancière, 1983: 43)

Así pues, la ignorancia y la pereza, ambas de la mano, son remedios inigualables a la hora de evitar que las almas de bronce pretendan imitar a las almas de oro, ejerciendo tareas que no son las propias de su naturaleza. Rancière lleva esta tesis más lejos (1983:73): la apelación a las distintas clases de "naturaleza" es para el filósofo-rey un modo tajante de evitar que el artesano pretenda ejercer la filosofía, pues inmediatamente se convertiría en un sofista, el penúltimo escalafón que puede encarnar un alma en la jerarquía de las encarnaciones. ${ }^{8}$ El buen artesano, por tanto, no será aquél que tenga un buen dominio de su oficio sino aquél que se limite exclusivamente a realizarlo. La pereza lo persuadirá de querer emprender otras labores, e incluso, de querer perfeccionarse en aquella que le corresponde según su naturaleza: "Con el derecho a la pereza, el artesano reconquista un cierto derecho a la mentira. [...] El zapatero no debe ser otra cosa que zapatero. Pero no está obligado a serlo verdaderamente" (Rancière,1983:43). Ahora bien, si el filósofo tiene derecho a la mentira, y en el contexto de esta interpretación podríamos hasta considerarla una parte de su deber como gobernador, no tiene, sin embargo, derecho a la pereza (vid. Rancière, 1983: 78) ${ }^{9}$. La mentira a la que el filósofo se ve forzado no es, en absoluto, de la misma clase

6 Traducción propia.

7 Traducción propia.

8 Para la jerarquía de las encarnaciones, según la sabiduría del alma, cfr., Platón, Fedro (248b-c).

9 Rancière interpreta de este modo el pasaje del Fedro en el que Sócrates sugiere que no es propio de un hombre sino de esclavos dejarse adormecer por el sol del mediodía y el canto de las cigarras. Cfr. Fedro, (259 a-b) 
que la mentira del artesano que puede permitirse serlo solo en apariencia. Por su parte, el guardián no comparte ni con el artesano el privilegio de la pereza, ni con el filósofo-rey el deber del engaño ${ }^{10}$. En la interpretación de Rancière esto forma parte de la jerarquía de las funciones sociales, según la cual, unas tareas son más valiosas que otras y, justamente aquellas que son fruto de lo superfluo (el oficio militar producto del lujo, y la filosofía que se vuelve necesaria para educar a los guardianes y distinguir la naturaleza de las almas) terminarán por encabezar la jerarquía, mientras que las tareas de artesanos, agricultores y comerciantes, de primera necesidad, compondrán la base de la pirámide. Al mismo tiempo, otra clase de tareas relacionadas con el "régimen estético de las artes", serán completamente prohibidas dado que suponen una amenaza para el régimen policial que Platón plantea. Así entiende Rancière la prohibición de los poetas en la ciudad ideal platónica, pues estos rompen deliberadamente con el principio monotécnico, al confundir en el ámbito de la ficción, roles, identidades y lugares. "La proscripción platónica de los poetas se funda en la imposibilidad de hacer dos cosas al mismo tiempo. El problema de la ficción es primero un problema de distribución de lugares" (Rancière, 2009:11). En general, toda tarea mimética es severamente condenada y excluida del régimen platónico, pues infringe el imperativo de la monofunción (vid. Rancière, 2009: 53-54). Por el contrario, los oficios del filósofo y el guardián, situados en la cúspide de la pirámide social, suponen un quehacer muy serio a efectos sociales, y por ello, un filósofo y un guardián sí están obligados a serlo realmente y no solo a parecerlo; en cambio, el oficio de artesanos, agricultores y comerciantes, en la base de la pirámide, debe simplemente mantenerse constante, incluso si no se lleva a cabo más que de una forma superflua y sin mayor afán de perfección. Es así como Rancière llega a la conclusión de que lo superfluo corrige el peligro de lo necesario, pues los oficios nacidos de lo superfluo (del lujo y de la guerra) refrenan el posible exceso en el que podrían incurrir aquellos que desempeñan las funciones más necesarias. Pero es preciso matizar que si lo superfluo corrige este peligro, lo hace a través del derecho parcial a la pereza que esta élite del excedente otorga a las bases sociales. La pereza como derecho compone el núcleo del derecho a la apariencia, y en términos de Rancière, del derecho a la mentira. Apariencia, mentira y pereza van unidas cuando se trata de la apariencia del demos. Como Rancière expone en su obra política fundamental, El desacuerdo, en Platón los términos "demos" y "doxa" se asocian mediante una relación de sinonimia: "hubo doxa, "apariencia" para el pueblo, apariencia de pueblo" (Rancière, 1996: 23). El pueblo toma decisiones basándose en la doxa, en la apariencia que es el dominio en el cual le es legítimo vivir y desempeñar su monotecnia. Así es como un pueblo con derecho a la apariencia se convierte en "apariencia de pueblo", y al consentir este derecho, el gobernante permite que la ciudad ideal se impregne de una patología necesaria, la ademia ${ }^{11}$. Porque la apariencia de pueblo es la ausencia de pueblo. Pero esta ademia -término que rescata Giorgio Agamben- solo es posible si el pueblo consiente primero en dejarse aparentar, en dejarse invisibilizar, esto es, si el pueblo incurre en otro concepto trabajado por Agamben: la acedia. La acedia no remite solamente a una fatiga psíquica, sino ante todo a "la parálisis del ánimo" (Agamben,

10 "Pero también la embriaguez, la molicie y la pereza son por completo inapropiadas para los guardianes" Cfr. Platón, La República, traducción por Conrado Eggers, Madrid: Gredos. 1994. (398e)

11 Cfr. G. Agamben (2016). El fuego y el relato. Barcelona: Sexto Piso. P.59. 
2001: 33), la fuga del espíritu interruptor de lo policial. Y por tanto lleva implícito el consentimiento absoluto al reparto policial, y este consentimiento a su vez explica por qué un pueblo consiente en desaparecer al aceptar su derecho a la apariencia. A través de la acedia generalizada, garante de la monotecnia, la ademia se hace posible y en sus manos, la doxa se ejerce con total impunidad. La distancia entre el sabio y el ignorante queda desde entonces abierta por medio de esta continua división basada en la desigualdad entre quienes tienen y no tienen derecho a la apariencia. Acedia-ademia-impotencia son tres patrones sociales que van a repetirse a través de la historia para dar lugar a situaciones políticas y culturales similares. Porque en términos de Rancière, ahí donde el tiempo nos iguala, la historia nos divide. Rancière va a definir la historia como "la pertenencia de los sujetos a un mismo tiempo" (2012: 18), y no obstante, siguiendo la lógica de la desigualdad, habrá siempre en todas las épocas seres históricos, seres que hacen historia, frente a aquellos que solo tienen derecho a ejercer su función anónima en tanto "seres de tiempo", sin permiso para entrar en "el orden de la memoria". De este modo, el tiempo pone de manifiesto la contingencia de la igualdad y viene a interrumpir el repertorio policial que la historia, una y otra vez, en todos los tiempos, administra y selecciona.

\section{Por qué el "maestro ignorante" no es ignorante}

\section{El que no puede mandarse a sí mismo debe obedecer. (F. Nietzsche).}

Platón entiende que la pereza es propicia dado que impide que las naturalezas inferiores traten de imitar a las superiores. En este sentido, la pereza se considera una falta de voluntad necesaria y beneficiaria a efectos sociales porque garantiza la monotecnia, fundamento de la ciudad ideal. Contra esto, Rancière defiende que el mito platónico de las jerarquías de las naturalezas es una artimaña de la que se vale el filósofo-rey para garantizar su posición de poder y su posición de saber. Valiéndose del ejemplo histórico de Joseph Jacotot y de su experimentación pedagógica, Rancière va a postular contra Platón la igualdad de las inteligencias, la isonousía, como base de hecho de un orden social desigual. La isonousía viene dada por naturaleza, mientras que la naturaleza de la sociedad es instaurar una desigualdad artificial. Entre el modelo platónico y la teoría de Rancière, vamos a cruzar rápidamente la delgada línea que separa a la monotecnia de la pantecnia. En la esfera de la pedagogía, el mito platónico de la jerarquía de las naturalezas se traduce en el mito de la explicación que levanta la frontera entre los maestros "explicadores" y los alumnos "explicados" (Rancière,2003: 9). El método Jacotot -también llamado: "método de la igualdad" o "método de la voluntad"- trabaja sin explicaciones, dado que no cree en la desigualdad de las capacidades intelectuales, y enseña cómo un emancipado puede enseñar a otros a emanciparse sin necesidad de explicaciones. Como Rancière no se cansa de repetir, en este método de enseñanza universal no se trata de que los alumnos aprendan pintura, piano, literatura o matemáticas, sino de que aprendan que pueden aprenderlo solos, es decir, que sean conscientes de la igualdad de las inteligencias. Por tanto, la persona que ejerce el papel de maestro emancipador solo ha cumplir un único requisito: estar él mismo emancipado. No ha de saber aquello a través de lo cual enseña a otros a emanciparse, ya sea pintura, literatura, matemáticas, etc. De aquí en adelante, habrá que poner atención a la noción misma de "maestro 
ignorante" que maneja Rancière y al enunciado según el cual, los alumnos aprenden del maestro lo que éste ignora (vid. Rancière, 2003:12 y 59). Caemos en terrenos ambiguos y dados a los juegos de palabras y a los roces difusos de sentido. Lo que el maestro emancipado busca enseñar es la emancipación, y para ello él mismo debe estar emancipado: luego el maestro no ignora lo que enseña, lo que ignora es la disciplina a través de la cual su método va a hacerse efectivo. El objeto de enseñanza es la emancipación y el maestro ha de estar emancipado para enseñarla. Aunque esta enseñanza no se lleve a cabo por medio de explicaciones, la lógica del "orden explicador" según la cual el que enseña sabe más que el que aprende no se ve rota por el método de Jacotot. De entrada, el maestro emancipador sabe que no sabe más que el alumno, pero al saber eso ya está sabiendo más de lo que el alumno sabe. El hecho de que el profesor explicador o emancipador sepa más que aquel que aprende no quiere decir, sin embargo, que sea más inteligente, pero sí hay, de modo evidente, una distancia natural que separa al profesor del alumno en un primer momento. Ningún agente externo puede "auto-emanciparnos", luego para llegar a ser conscientes de la igualdad de las inteligencias necesitamos sobre nosotros el peso de una voluntad que fortalezca la nuestra. Por tanto, este método encierra una paradoja ambigua: cuánto más quiere demostrar la igualdad de las inteligencias más está obligado a admitir la desigualdad de las voluntades. Someter la voluntad del alumno, verificar su atención y vigilar su búsqueda son los tres pasos fundamentales de este método.

Exigencia incondicionada: el padre emancipador no es un pedagogo bonachón, es un maestro intratable. El mandato emancipador no conoce tratados. Ordena completamente a un sujeto al que supone capaz de ordenarse él mismo. (Rancière, 2003: 24)

Al postular la isonousía, la diferencia existente entre el maestro y el alumno ha de recaer necesariamente en la voluntad. "El hombre es una voluntad servida de una inteligencia", nos dice Rancière (2003: 31). Así pues, la voluntad del maestro debe ser férrea a la hora de guiar al alumno hacia la emancipación. En este sentido, la distancia que separa a ambos, profesor y discípulo, no es una distancia que pueda explicarse en términos de mayor o menor capacidad intelectual, sino en términos de mayor o menor voluntad, es decir: de mayor o menor pereza. Desde esta óptica, la pereza está destinada a ser la pasión más condenable de todas, único factor que puede darle la razón a la sinrazón del orden explicador que divide jerárquicamente el mundo de las inteligencias.

"No puedo" es así una frase de olvido de sí mismo, de donde el individuo razonable se ha retirado. [...] Nadie está en el error si no es por maldad, es decir, por pereza, por deseo de no oír hablar más de lo que un ser razonable se debe a sí mismo. [...] el mal está en divagar, en salir del propio rumbo, en no prestar ya atención a lo que se dice, en olvidar lo que se es. (Rancière, 2003: 34)

Siguiendo la tendencia racionalista, presente en todo el discurso de Rancière, los seres emancipados son seres"razonables" dado que "el ser racional es ante todo un ser que conoce su potencia, que no se engaña sobre ella" (Rancière, 2003:34). Por tanto, los seres razonables son aquellos que no se han olvidado a sí mismos y han empleado su voluntad para redirigir 
y avivar el rumbo de su inteligencia por medio del autoaprendizaje. Los seres razonables han superado la sordera, el uberhören del que habla Heidegger como una de las posibilidades esenciales del oír ${ }^{12}$. Los no-emancipados, en este sentido, son aquellos que no desean "oír hablar más", que no prestan "ya atención a lo que se dice". Por tanto, tenemos como punto de partida la igualdad de todos los seres humanos a la hora de poder comprender, de poder oír, y también la desigualdad de los mismos a la hora de querer comprender, de querer oír. Con esto, nos situamos en el postulado heideggeriano de que "pensar es oír y ver" (Derrida,1998: 376). Aquellos que no oyen, que oyen mal, han tomado el camino fácil dado que "es más fácil ausentarse, ver la mitad, decir lo que no se ve, decir lo que se cree ver" (Rancière,2003: 33) A través de la pereza, los seres humanos ven y oyen tan solo a medias, se abandonan a sí mismos, y con ellos el poder de sus inteligencias. La pereza se convierte así en el primer defecto y en el más letal de todos porque "es el defecto de la voluntad lo que hace errar a la inteligencia".(Rancière, 2003: 33) No se trata ya de una desigualdad en el ver, en el oír, y por añadidura en el pensar, sino una diferencia en el querer-ver, querer-oír, querer-pensar. Así pues, si en la ciudad ideal de Platón los guardianes y los filósofos no podían permitirse la pereza, en el sistema ideal de la enseñanza universal nadie puede permitírsela si pretende alcanzar su emancipación. De este modo, Rancière explica cómo los genios no pueden permitirse la pereza, dado que la genialidad se funda en el esfuerzo incansable de la voluntad y no en la superpotencia intelectual del genio con respecto a sus semejantes. El oído que Rancière busca afinar, por medio del método Jacotot, tiene cierto aire de familia con "el oído poético" del que nos habla Heidegger, el oído que se mantiene firme en la escucha "del origen por el que tiene pasión" (Derrida, 1998: 372). No es casualidad que el planteamiento de Rancière concluya que una sociedad de iguales debería traducirse en una comunidad de artistas, de "espíritus activos: hombres que hacen, hablan de lo que hacen y transforman así todas sus obras en modos de significar la humanidad [...]" (2003: 41). En el seno de esta comunidad, "la inferioridad de alguien es consecuencia de la circunstancias que no le obligaron a seguir buscando" (2003: 41). El que quiere oír es aquel que se mantiene firme en la escucha de sus propias capacidades y competencias, pero es también el que desoye la voz de la desigualdad que emana de la fuente social. Hasta ahora, hemos visto que el maestro "ignorante" enseña al alumno a trabajar lo que él ya ha trabajado de antemano: la voluntad. En este sentido, no puede considerárselo ignorante. A continuación pasamos a analizar el precio a pagar por esta igualdad de las inteligencias, a saber: la desigualdad volitiva del alumno con respecto al maestro.

\section{Igualdad intelectiva y desigualdad volitiva: la pereza en el orden explicador}

La meta que persigue un maestro emancipador al estilo de Joseph Jacotot, podría ser explicada mediante la reflexión que María Zambrano hace sobre su experiencia como discípula de Ortega y Gasset:

La acción del maestro trasciende el pensamiento y lo envuelve; sus silencios valen a veces más que sus palabras y lo que insinúa puede ser más eficaz que lo que expone 12 "Así pertenece a lo propio del oír el que precisamente pueda el hombre oír mal (desoír) en tanto que desoye lo
esencial"; Jacques Derrida (1998). El oído de Heidegger. Barcelona: Trotta. P.375. 
a las claras. Si hemos sido en verdad sus discípulos, quiere decir que ha logrado de nosotros algo al parecer contradictorio; que, por habernos atraído hacia él, hayamos llegado a ser nosotros mismos. (1982: 93)

El maestro emancipado al enseñarnos lo que posee -la emancipación- a través de lo que no sabe, nos enseña que siempre habíamos podido saber por nosotros mismos aquello que gracias a él aprendimos. Atrayéndonos hacia él nos sumerge en la experiencia de nuestra propia capacidad para aprender cualquier cosa que nos propongamos. Pero hasta cierto punto, el maestro emancipado corre el mismo peligro que Rancière denuncia en el "atontamiento perfeccionado" llevado a cabo por Sócrates. Pues si Sócrates guía al alumno por el camino y hacia el destino que el mismo Sócrates conoce de antemano, -dejando al aprendiz con la impresión de que jamás hubiera aprendido de no haber sido ayudado por Sócrates-, el maestro emancipado, cuya voluntad es más poderosa que la de su discípulo, también conduce al alumno hacia un estado que él mismo conoce de antemano y aunque se valga de otros métodos, difícilmente el alumno emancipado podrá dejar de sentir una deuda con aquel cuya lucidez volitiva es superior a la suya. Terminado su trabajo emancipador, el maestro de la enseñanza universal podría retirarse, pues el alumno tiene en sus manos, actualizados, todos los recursos posibles para adquirir cualquier saber. Lo que hará a partir de entonces el alumno emancipado será aplicar la voluntad que ha trabajado sobre su propia inteligencia y compartir esta fuerza de voluntad con aquellos que todavía están inmersos en el juego de la desigualdad: el juego de la incapacidad y la pereza. Pero la pereza también ha sido fuertemente atacada por aquellos que, en el ámbito educativo, están lejos de defender la isonousía. En el orden explicador, la pereza está tan denostada como en el orden emancipador; tampoco en esto pueden diferenciarse los dos órdenes.

El filósofo y teólogo español, Jaime Balmes, en una de sus obras pedagógico-filosóficas más célebres, El criterio, considera a la pereza "la pasión de la inacción" dado que "tiene para triunfar una ventaja sobre las demás pasiones y es el que no exige nada; su objeto es de una pura negación[...] Todas las pasiones para el logro de su objeto exigen algo; solo la pereza no exige nada" (1940: 116). Siendo contemporáneo de Joseph Jacotot, Jaime Balmes está lejos de secundar la igualdad de las inteligencias, y a pesar de su énfasis en la necesidad de ejercitar el intelecto para conocer el límite y el alcance de sus capacidades, sus indicaciones parecen situarse en la línea establecida del orden explicador. Apuesta, más bien, por una proporción desigual de las capacidades: "La enseñanza tiene dos objetos: primero, instruir a los alumnos en los elementos de la ciencia; segundo, desenvolver su talento para que al salir de la escuela puedan hacer los adelantos proporcionados a su capacidad" (Balmes, 1940: 71). Con todo, ambos órdenes encuentran en la pereza y la distracción sus mayores obstáculos. Así, según Rancière: "El pecado original del espíritu no es la precipitación, es la distracción, es la ausencia" (2003:33). Y también para Balmes la atención es el primer recurso de todo aprendizaje ordenado: "El primer medio para pensar bien es atender [...]Con la atención notamos las preciosidades y las recogemos; con la distracción dejamos, quizá, caer al suelo el oro y las perlas como cosa baladî" (1940: 22). Un siglo antes que Balmes, John Locke ya afirmaba que la pereza "no puede ser nunca un resorte de acción" (2012: 176). Las teorías educativas de Locke son consideradas hoy como un preámbulo de la psicología pediátrica y ponen el énfasis en la importancia de educar y ejercitar el entendimiento humano desde 
edades tempranas. Incentivar en el niño la asociación de ideas es importante en el sistema de Locke, y en esto podríamos tender un modesto puente hacia el método Jacotot, el cual lleva al extremo la búsqueda de asociaciones y la considera, según Rancière, el núcleo de su método de enseñanza. Este eje asociativo en el que se apoya el método Jacotot consiste en "aprender alguna cosa y relacionar con ella todo el resto según este principio: todos los hombres tienen una inteligencia igual" (2003: 14). Pero esta no es la clase de conexión que Locke propone, a través de una propuesta empirista que rechazará el innatismo cartesiano, propio de su época. La asociación de ideas, en la mente de un niño concebida al modo de una tabula rasa, debe inscribirse conforme al modelo causa-efecto de las matemáticas. La mente sería así estructurada desde una edad temprana, para ampliar las capacidades mentales de los individuos. Éste es también el método que Locke propone a los hombres adultos que busquen ampliar su capacidad de raciocinio:

En primer lugar, porque [las matemáticas] convencen experimentalmente al hombre de que, para razonar bien, no basta con tener unas facultades que les satisfagan y les sirvan en su vida cotidiana. Con estos estudios [matemáticos], un hombre se dará cuenta de que aunque piense que su entendimiento es muy capaz, sin embargo le falla en algunas cosas, a veces de un modo muy visible. De este modo se desarraigaría esa presunción que la mayoría de los hombres tienen sobre sí mismos; y no estarían dispuestos a pensar que su mente no necesita ayuda para crecer y que nada podría añadirse a la agudeza y penetración de su entendimiento. (2012: 295)

Para Locke, como para Jacotot y para Rancière, los seres humanos somos por naturaleza racionales. No obstante, la idea de Locke es que la razón se atrofia sin el debido entrenamiento y aunque esta pérdida no sea irreversible, recuperarla a una edad adulta resulta una tarea laboriosa (vid. Locke, 2012: 292-293) Así, la supuesta facilidad con la que un padre de familia analfabeto podría enseñar "lo que ignora" (habiendo realizado antes, él mismo, la experiencia de aprender algo de manos de un ignorante; es decir, habiéndose ya emancipado), tal y como Rancière nos plantea, no solo parece anti-intuitiva, sino un camino extremadamente largo y arduo, y por tanto un sistema en el que la pereza tiene que ser, a la fuerza, un obstáculo aún más peligroso -por ser más probable su aparición-, que en el curso fraccionado del sistema explicador. Hay, además, un pensamiento nuclear del planteamiento educativo de Locke que contradice el corazón del proyecto universal de Jacotot: "El que no haya contraído el hábito de someter su voluntad a la razón de los demás cuando era joven, hallará gran trabajo en someterse a su propia razón cuando tenga edad de hacer uso de ella" (Locke,2012: 68). Ésta sería, precisamente, la definición de "atontamiento" según Rancière, es decir, la subordinación de una inteligencia humana a otra inteligencia humana. Sin embargo, la emancipación se articula en un proceso mediante el cual, el alumno somete su voluntad a la voluntad del maestro pero nunca su propia inteligencia a la de ningún otro ser humano (vid. Rancière,2003: 11-12). Pero Locke nos habla de someter la voluntad a la inteligencia (a la "razón") del maestro, y además, indica que esto debe acontecer a una edad temprana so-pretexto de que no hacerlo conlleve unas consecuencias fatídicas casi irreversibles. El hecho de que nuestra voluntad aprenda a someterse a la razón de los otros, (aprenda a obedecer a la inteligencia de un tercero), es fundamental para que un día nuestra 
inteligencia pueda mandar sobre sí misma. El sometimiento, en este contexto lockeano, es el primer paso de la emancipación. Partir de la negación de una inteligencia a obedecer a otras inteligencias y voluntades, implica confundir la meta con el punto de partida.

Si el "atontamiento" pasa por confiar nuestra inteligencia a otra inteligencia -que va a instruirla y a darle forma-, no está claro cómo confiar nuestra voluntad a otra voluntad -que va a instruirla y a darle forma-, es sinónimo de "auto-emancipación". Ya hemos dicho que la emancipación, para ser "auto", no puede provenir de fuentes externas. Sin embargo, Rancière, finalizando la obra, nos dice: "Solo un hombre puede emancipar a otro hombre" (2003: 56), y con esto queda descartado que el proyecto Jacotot apunte como meta a la autoemancipación -para la cual sería necesario que la figura misma del maestro, fuese del tipo que fuese, cayese por su propio peso-. En cambio, la emancipación que nos propone el método universal viene provocada y trabajada por agentes externos al alumno, y se relaciona con un sometimiento extremo de la voluntad del alumno a la voluntad del maestro. Precisamente porque se evita la sumisión de su inteligencia, la voluntad del alumno se ve sometida doblemente. "Llamemos atención al acto que pone en marcha a esa inteligencia bajo la presión absoluta de una voluntad" (2003: 18). Dicho de otro modo, el presupuesto de la igualdad de las inteligencias (isonousía), solo da lugar a la emancipación del alumno cuando el maestro presupone, obligatoriamente, la desigualdad de las voluntades (de aquí en adelante: "anisothelema"; aniso "desigual"; thelema: "voluntad"). El maestro emancipador no administra un conocimiento cerrado. En su lugar, impone una voluntad férrea. Tengamos en cuenta que el método educativo planteado por Rancière no cuestiona en ningún momento la necesidad de la figura del maestro. Esto lo advierte lúcidamente Gert Biesta, cuando entiende el proyecto planteado por Rancière como "un proceso en el que los profesores y sus enseñanzas son indispensables" (2017:53). El maestro es necesario como figura emancipadora, en cambio, puede ser nocivo como figura explicadora. Y lo que se opera en el cambio de un maestro explicador a uno emancipador, es el traslado de la sumisión de la inteligencia a la sumisión de la voluntad. Ahí donde se presupone una igualdad intelectiva, se exagera, forzosamente, una desigualdad volitiva. Porque si hacemos convivir en un mismo escenario, la igualdad de las inteligencias (isonousía) y la igualdad de las voluntades (isothelema), el maestro ya no conserva ningún rasgo que lo caracterice frente al alumnado. En este tipo de escenario pedagógico, la figura del maestro tendría que ser seriamente cuestionada, en tanto un igual entre iguales, un estudiante entre estudiantes, o un maestro entre maestros. Este planteamiento se acerca al proyecto educativo planteado por Paulo Freire, que revisaremos al final de este ensayo, pero no encaja con la propuesta de Rancière. Biesta (2017:62) explica esa diferencia entre inteligencia y voluntad, como una "disociación entre enseñar y comunicar", que sería crucial según este autor, en los planteamientos de Rancière. El maestro emancipador no comunica un saber, es más, lo que se quiere probar con el método Jacotot es que la enseñanza no consiste en comunicar un conocimiento específico. El acto de comunicar está emparentado con la desigualdad de las inteligencias, -está vinculando a la explicación-, mientras que el proceso de enseñar estaría ligado al sometimiento de la voluntad.

[...] lo que hizo Jacotot no fue reemplazar la inteligencia de sus alumnos con la suya propia, sino más bien, exhortarlos a usar su propia inteligencia. Por tanto, la relación 
entre Jacotot y sus alumnos no se basa en una relación de inteligencia a inteligencia, sino "de voluntad a voluntad" (Biesta, 2017:62)

Según Biesta, la innovación de Rancière consistiría en presuponer la igualdad, aquí y ahora, como punto de partida, y trabajar "como si" ésta ya viniese dada, y no tuviese que ser construida (Biesta,2012: 24; Biesta, 2017:64). No obstante, ni Biesta ni Rancière enfatizan el hecho de que la jerarquía de las igualdades se deshace en favor de otra jerarquía, a saber: la de las voluntades. Por ello, en nuestra opinión, habría que reformular el alcance de la innovación de Ranciére: su aportación principal consiste en establecer como punto de partida una clase de igualdad (la de la inteligencia: isonousía) y otra clase de desigualdad (la de la voluntad: anisothelema); en consecuencia, la ignorancia deja de ser una enemiga de la razón y la pereza pasa a ocupar el trono de las rivalidades. Y esta nueva jerarquía, en la que recae todo el peso y la dinámica pedagógica de la educación liberadora, hace que el método Jacotot no rompa en lo esencial con la estructura del orden explicador. Se requiere, en primer lugar, la intervención de una figura externa (el maestro) para lograr el despertar de la propia inteligencia; en ese proceso hay una desigualdad de base (la voluntad del maestro frente a la de los alumnos) que se impone en aras de una igualdad de base (la de la inteligencia del maestro frente a la de los alumnos), y como tal, el enemigo principal de esta nueva clase de emancipación ya no es la ignorancia, sino la pereza. Por un lado, si el papel del maestro fuese corregir la ignorancia de sus alumnos, ya no podríamos hablar de igualdad de inteligencias. Por otro lado, si el papel del maestro consiste en corregir la pereza de sus estudiantes, no podemos hablar de igualdad de voluntades. No se puede salvaguardar la figura del maestro si ninguna clase de desigualdad interviene en la ecuación; la figura del profesor exige de algún desnivel que justifique su posición y su función frente a los otros. Si esta desigualdad no se produce en el plano del conocimiento, ni tampoco en el plano de la voluntad, su tarea entra en crisis. Por otro lado, si mantenemos la desigualdad de las voluntades, tal y como hace Rancière, ¿en qué momento el alumno, sometido a "la presión absoluta de una voluntad", podrá sentir que se ha emancipado sin ayuda? ¿dónde queda la auto-emancipación en el contexto de la sumisión a un maestro "intratable"? Además, Rancière establece una asociación entre el cumplimiento de la condición racional y la voluntad, "el aprecio de sî", mientras que la pereza, en tanto “olvido de sî́, parecería no hacer justicia a la racionalidad inherente al ser humano.

Por voluntad entendemos esta vuelta sobre sí del ser racional que se conoce actuando. Es este foco de racionalidad, esta conciencia y este aprecio de sí como ser razonable en acto lo que nutre el movimiento de la inteligencia. (Rancière 2003: 34)

Aquí volvemos a toparnos con una asociación en términos logocentristas: la voluntad responde al orden de la racionalidad (el logos), la pereza, en cambio, lo infringe y lo traiciona. Cabe resaltar aquí una de las dimensiones que entraña esta apuesta por la voluntad férrea del maestro, a saber: el hecho de que Rancière parece dejar completamente de lado la esfera de los afectos en el proceso emancipador. Efectivamente, al describir al maestro como una "figura intratable", el filósofo francés confirma la carencia afectiva que nos encontramos en su planteamiento pedagógico. Podría parecer que esta carencia juega un 
papel activo y decisivo en la función del maestro, en un escenario en el que la inteligencia emocional brilla por su ausencia, y el sometimiento volitivo se reivindica como emblema sine qua non de la racionalidad. El ser emancipado es un ser racional, ha sometido su voluntad a la de un maestro con el fin de iluminar el potencial de su inteligencia. Dado que en este proceso las emociones no toman parte, entendemos que esta emancipación no tiene por qué derivar en una emancipación al nivel de los afectos, sino solo al nivel de la razón. El enfoque es logocentrista, y sus efectos también. En un proyecto educativo en el que todo recae sobre la sumisión de la voluntad, la consideración de la esfera emocional de los estudiantes podría ser tan nociva como lo es la multitarea de los individuos para el régimen político de Platón.La conjunción de este binomio igualdad/desigualdad (isonousíalanisothelema), tomado como punto de partida y presupuesto, lleva implícito un doble movimiento, externo e interno, por parte del alumno. De un lado, la purificación de toda aprehensión distraída y perezosa instalada en su ánimo y en su mente, lo cual requiere someterse a un maestro con una voluntad más vigorosa que la suya (anisothelema); de otro lado, un empoderamiento de sus capacidades intelectivas que no pueden caer en la misma sumisión a la que se entrega su voluntad, pues en el ámbito de la inteligencia, alumno y maestro son iguales (isonousía).

\section{Conclusiones}

Tras denunciar la monotecnia que Platón postula en La República como un medio para lograr el orden y la estabilidad social, Rancière abre las puertas, discretamente, a la posibilidad de la pantecnia. A pesar de que él mismo declara que la isonousía no desemboca en la máxima: "quien quiere puede", tampoco procede a resolver esta posible objeción sino que se limita a rechazarla. Paradójicamente, Rancière nos explica en qué consiste el método de la no explicación y, de hecho, va a llevar la isonousía hasta el límite del potencial artístico de todo ser humano. De la misma manera que no se trata de "crear sabios" sino de "crear emancipados", tampoco en un terreno como el de la pintura, se tratará de crear "pintores" sino de "crear emancipados" (vid. Rancière, 2003: 38-39). En concreto, se trata de crear seres emancipados que se atrevan a decir "yo también soy pintor". Esta contradicción evidente obliga a Rancière a redefinir la acepción comúnmente aceptada de "pintor": "Yo también soy pintor significa: yo también tengo un alma, tengo sentimientos para comunicar a mis semejantes" (2003: 39). De este modo, cualquiera que se atreva a expresarse mediante la pintura, pasa a ser un pintor. Estos "emancipados" en calidad de "artistas" no tienen la obligación ni el objetivo de crear obras maestras, nos dice Rancière; usan el arte para expresar y compartir su emancipación. Rancière no solo cae en una concepción logocentrista del arte, algo que ya señalamos en la introducción, sino que, discretamente, lo tiñe de un hálito pragmatista. El arte sería una práctica emancipadora -cuya utilidad es servir al proceso emancipador- y una forma de verificar, asimismo, la correcta emancipación de quien se llama a sí mismo "pintor" por tener emociones que transmitir en calidad de ser razonable. Esta consideración está próxima a una esfera particular del arte contemporáneo, donde la mera intención de transmitir por parte de un sujeto es condición suficiente para que éste devenga artista y para que las obras resultantes de tal proceso -pictóricas, escritas, audiovisuales, etc.- sean catalogadas como "obras de arte". 
En lo que respecta a la crítica de un mundo institucional dividido en dicotomías artificiales del tipo sabio-ignorante, superior-inferior o competente-incompetente, los planteamientos de Rancière guardan ciertas similitudes con las de Paulo Freire. En la célebre obra de este teórico de la educación, titulada La pedagogía del oprimido, encontramos conceptualizadas de otra manera, y en el seno de un estudio sociológico, las mismas ideas que expresa Rancière al hablar del "orden explicador". Sin embargo, en la obra de Freire, el "orden explicador" se designa como proyecto de "educación bancaria" mientras que una educación cuya meta fuese la emancipación de los individuos se denominaría "educación problematizadora" o "educación libertadora".

En la visión "bancaria" de la educación, el "saber", el conocimiento, es una donación de aquellos que se juzgan sabios a los que juzgan ignorantes. Donación que se basa en una de las manifestaciones instrumentales de la ideología de la opresión: la absolutización de la ignorancia, que constituye lo que llamamos alienación de la ignorancia, según la cual ésta se encuentra siempre en el otro. (Freire, 1992: 77)

El hecho de que la distancia artificial entre profesor y alumno sea fruto de un constructo institucional al que Rancière llama “orden explicador" y Freire "educación bancaria”, ha sido denunciado por ambos autores en contextos diferentes, siguiendo líneas de argumentación muy similares. No obstante la educación libertadora de Paulo Freire propone que la relación "educador-educando" sea de completa igualdad, esto es, dialógica. El educador se identifica, según esto, con los alumnos y su acción

debe orientarse en el sentido de la liberación de ambos. [...] Su acción debe estar empapada de una profunda creencia en los hombres. Creencia en su poder creador. Todo esto exige que sea, en sus relaciones con los educandos, un compañero de éstos. (Freire, 1992: 82)

En cambio, el maestro emancipador que Jacotot propone y que Rancière enfatiza, debe presionar absolutamente la voluntad del alumno, y está obligado a convertirse en un "maestro intratable", por lo que la educación emancipadora no puede llevarse a cabo en términos de compañerismo o incluso en términos de diálogo. Gert Biesta hace hincapié en esta diferencia crucial entre el enfoque de Freire y el de Rancière, dado que el primero "deja al maestro fuera de la ecuación" (Biesta: 2017:64), mientras que el segundo le atribuye un rol esencial en el proceso de emancipación. Efectivamente, Freire arremete contra la figura autoritaria del profesor, al que considera la piedra angular de la educación bancaria. Curiosamente, el enfoque de Rancière necesita de esta figura "intratable", férrea, autoritaria, que sepa imponer su voluntad sobre aquellos que lo igualan en inteligencia. En nuestra opinión, la figura del maestro es vital en el proyecto de Rancière porque su propuesta solo afecta a una dimensión de la igualdad (la intelectiva), al contrario que la propuesta de Freire, más ambiciosa en este sentido. Esto explicaría también otra diferencia importante entre ambos enfoques, señalada por Biesta (2017:64): el hecho de que Rancière pueda permitirse dar por hecho la igualdad (intelectiva), tomándola como punto de partida, mientras que la igualdad planteada por Freire (integral, operativa en todos los niveles) tiene que ser proyectada en 
términos de un ideal y una meta futura. Pero la distancia definitiva que se levanta entre ambos planteamientos viene dada por el campo de acción al que se dirigen. No es un detalle menor el que Rancière espere hasta el final de su obra para anunciar que el método de la igualdad no debe extenderse al ámbito de la sociedad, sino que debe ceñirse al perímetro de lo individual. Así, Freire nos habla de una emancipación como proceso educativo dialógico (de completa equidad entre las partes), que tiene como finalidad combatir la desigualdad en un futuro, y plasmar la igualdad en el área social-institucional. Por el contrario, Ranciére problematiza la emancipación como una posibilidad ya presente, presuponiendo un cierto tipo de igualdad, pero este proceso de "enseñanza universal" no aspira a ser el programa educativo del conjunto de la sociedad. Los individuos son racionales, nos dice Rancière, pero las sociedades no. Por tanto, el lugar del método Jacotot y el lugar de la emancipación está en la individualidad de la conciencia, y no en el estrato policial de las instituciones. Tener en cuenta este contexto individual en el que se enmarca el proyecto de Rancière, es vital para entender su propuesta, según Biesta: "el argumento de Rancière es un argumento sobre la emancipación y el rol del maestro en la educación emancipadora, y no una teoría general de la educación, la escolaridad, o las dinámicas de instrucción (didácticas)" (2017: 63). No coincidimos con Biesta, cuando denuncia la interpretación errónea de la que es objeto el proyecto de Rancière, en el ámbito de la filosofía de la educación. A nuestro entender, es la propia obra la que invita, durante largos intervalos, a una interpretación que rebasa el área de lo particular. Conviene recordar que lo que Rancière denuncia es una desigualdad artificial en el ámbito de lo general y por ello no es de extrañar que su planteamiento haya sido entendido "como una discusión general acerca de la educación-como-instrucción, y no como una discusión específica de la educación-como-emancipación” (Biesta, 2017:65). En cierto sentido, podría decirse que Rancière nos deja con un problema de cuño social (general) y una propuesta a nivel individual (particular), que no podría ser aplicada en ningún caso al dominio universal del problema. Al menos, así concluye este filósofo sus investigaciones acerca del método emancipador, habiendo logrado extender un concepto central de su obra filosófica, la igualdad, al ámbito pedagógico. No sin levantar polémica, ni sin dejar cabos sueltos. Como hemos tratado de mostrar en este ensayo, Rancière se ve obligado a sacrificar una dimensión de la igualdad (la volitiva) pasa salvaguardar la equidad intelectiva, y finalmente, restringe el alcance de su planteamiento al campo del individuo.

\section{Referencias}

Agamben, G. (2016). El fuego y el relato. Barcelona, España: Sexto piso.

Agamben, G. (2001). Estancias.Valencia, España: Pre-textos.

Balmes, J. (1940). El criterio. Madrid, España: Ediciones Ibéricas.

Biesta, G. (2017). «Don't be fooled by ignorant schoolmasters: On the role of the teacher in emancipatory education», Policy Futures in Education, 15 (I), pp. 52-73. DOI: $10.1177 / 1478210316681202$.

Biesta, G. (2012). «Doing emancipation differently: transgression, equality and the politics of learning», Civitas Education is. Education, Politics and Culture, 1, 15-30.

Derrida, J. (1998). El oído de Heidegger. Barcelona, España: Trotta.

Derrida, J. (2001). La verdad en pintura. Barcelona, España: Paidós. 
Freire, P. (1992). La pedagogía del oprimido. (43a . ed.) Madrid, España: Siglo XXI. Locke, J. (2012). Pensamientos sobre la educación. Madrid, España: Akal. Rancière, J. (1983). Le philosophe et ses pauvres. París, Francia: Fayard. Rancière, J. (2012). Figures de l'histoire. París, Francia: PUF.

Rancière, J. (2010).El espectador emancipado, Buenos Aires, Argentina: Manantial. Rancière, J. (2003). El maestro ignorante. Barcelona, España: Laertes.

Rancière, J. (1996). El Desacuerdo. Buenos Aires, Argentina: Nueva visión. Rancière, J. (2009). El reparto de lo sensible. Santiago de Chile: LOM.

Zambrano, M. (1982). España. Sueño y verdad, Barcelona, España: Edhasa. 